\title{
EMPRENDIMIENTO Y PLAN DE NEGOCIO PARA INICIAR UNA HELADERÍA VEGANA EN EL CANTÓN PORTOVIEJO, ECUADOR
}

\section{ENTREPRENEURSHIP AND BUSINESS PLAN TO START A VEGAN ICE CREAM PARLOR IN THE PORTOVIEJO CANTON, ECUADOR}

\author{
Verduga-Pino Alexandra María ${ }^{1 *}$ \\ ${ }^{1}$ Doctora en Ciencias Económicas, Profesora Titular Universidad Técnica De Manabí. \\ Portoviejo - Ecuador. https://orcid.org/0000-0003-4864-0499 \\ *Correo: alexandra.verduga@utm.edu.ec \\ Resumen
}

\begin{abstract}
Se presentan los aspectos claves de un Plan de Negocios con aplicación práctica para pequeños emprendimientos, que a diferencia de los planes para medianas y grandes empresas exigen un nivel de preparación, recursos y tecnologías que representan seria ilimitaciones para la microempresa. Las alianzas con universidades y entidades locales de apoyo al emprendimiento facilitan la preparación de los planes de negocios. Teniendo como base las oportunidades identificadas en el mercado de helados artesanales en Ecuador y en particular en Portoviejo, así como las tendencias al consumo de productos más sanos. El objetivo del proyecto es la elaboración y venta de helados nutritivos y que no contenga ningún derivado o aditivo de origen animal, es decir helados veganos y para personas con intolerancia a la lactosa. Se realizaron estudios del mercado local, la valoración de los aspectos técnicos e identificación de los equipos necesarios para la inversión. Además, se elaboró el plan de mercadotecnia para el lanzamiento inicial y por último se validó desde el punto de vista económico financiero la factibilidad del proyecto. El mismo va a tener impactos en la diversidad comercial de la comunidad, el empleo femenino y la alimentación sana de la población, en particular las que tienen riesgos particulares de salud.
\end{abstract}

Palabras claves: plan de negocio, helados veganos, emprendimiento.

\begin{abstract}
The key aspects of a Business Plan with practical application for small enterprises are presented, which unlike plans for medium and large companies require a level of preparation, resources and technologies that represent serious limitations for microenterprises. Alliances with universities and local entities to support entrepreneurship facilitate the preparation of business plans. Based on the opportunities identified in the artisan ice cream market in Ecuador and in particular in Portoviejo, as well as the trends towards the consumption of healthier products. The objective of the project is the production and sale of nutritious ice cream that does not contain any derivative or additive of animal origin, that is, vegan ice creams and for people with lactose intolerance. Studies of the local market were carried out, the valuation of the technical aspects and identification of the necessary equipment for the investment. In addition, the marketing plan for the initial launch was prepared and, finally, the feasibility of the project was validated from the economic and financial point of view. It will have an impact on the commercial diversity of the community, female employment and the healthy diet of the population, particularly those with particular health risks.
\end{abstract}

Keywords: business plan, vegan ice cream, entrepreneurship.

Información del manuscrito:

Fecha de recepción: 22 de febrero de 2021.

Fecha de aceptación: 19 de abril de 2021.

Fecha de publicación: 23 de abril de 2021. 


\section{Introducción}

El negocio relacionado con la producción y comercialización de helados es muy amplio y variado, y se caracteriza por los constantes cambios e innovaciones. De acuerdo con Codex Alimentarius define helado comestible como todos los productos edulcorados obtenidos a partir de una emulsión de grasa y proteínas, con la adición de otros ingredientes y sustancias, o a partir de una mezcla de agua, azúcares, y otros ingredientes, y sustancias, que han sido tratados por congelación, y que se destinan a la venta, y consumo humano en estado de congelación o congelación parcial. (Codex Alimentarius, 2014)

En el caso del helado artesanal, constituye una preparación alimentaria que se obtiene de una mezcla de ingredientes, llevada a un estado blando y a la temperatura de consumo (aproximadamente de $-8 \mathrm{a}$ -13 grados centígrados), mediante congelamiento rápido y agitación simultánea que permite la incorporación moderada de aire. Generalmente se consideran y distinguen los helados a base de leche y helados a base de fruta y agua, definidos como sorbetes. El helado artesanal generalmente se prepara en el día, con ingredientes primarios y caracterizadores. Las mezclas para este helado se preparan con un método artesanal, generalmente con procedimientos "en caliente" para helados a base de leche, "en frío" para los sorbetes.

Una heladería común ofrece helados a base de productos lácteos, huevos, miel, harinas procesadas etc.; cosa que sucede también con sus acompañantes o hasta el mismo cono. Sin embargo, se ha identificado una oportunidad para innovar en un segmento de mercado que no se ha atendido adecuadamente en el territorio objeto de análisis, que es el cantón de Portoviejo en Ecuador. Para traducir la idea del negocio en un emprendimiento empresarial, independientemente del tamaño, requiere elaborar el plan de negocios para determinar la viabilidad de un proyecto empresarial desde el punto de vista mercadotécnico, marketing, operacional, organizacional y financiero., adaptado a las particularidades de una microempresa. 
La idea de negocio trata de la creación de una heladería vegana en la ciudad de Portoviejo. El veganismo es un término que data de los años 40, y fue acuñado por primera vez en 1944 por el británico Donald Watson. Un vegano es un sujeto que no ingiere productos alimenticios de origen animal. Al igual que los vegetarianos, los veganos no comen carne de ningún tipo (de cerdo, vaca, cordero, pescado, pollo, etc.) pero, a diferencia de los ovolactovegetarianismo, tampoco consumen huevos, lácteos ni miel (Pérez \& Merino, 2010). Entre los casos que se puede nombrar, son los consumidores intolerantes a la lactosa, es decir que no pueden digerir la azúcar de la leche y los productos lácteos, cosa que, si lo llegan a hacer, provocarían molestias como cólicos, hinchazón, gases, otros. Por otro lado, justamente también los ingredientes de los que están hechos la mayoría de conos de helados que hay en el mercado o los adherentes que se le ponen a los helados contienen lactosa, por lo que se pretende también atacar esta problemática.
Al ser un mercado casi inexistente en Manabí y en la ciudad Portoviejo, para este tipo de emprendimiento se requiere llevar a cabo el desarrollo del respectivo plan de negocio, para determinar los requerimientos para implantar el determinado negocio, si el producto a ofertar va a tener la acogida por los portovejenses, si es viable económicamente; pero principalmente si tiene posibilidades de crecimiento futuros.

El problema de investigación está referido a conocer si las bases del Plan de Negocios permitirán garantizar en la práctica, la sostenibilidad económica y financiera del proyecto considerando las acciones a proyectar ante los diversos escenarios que pudieran presentarse.

Los antecedentes para la elaboración del Plan de Negocios, en el tipo de emprendimiento que se proyecta, estuvo vinculado al estudio de factibilidad para la puesta en marcha de una heladería dedicada a la producción y comercialización de helados de yogurt con frutas "ice fruit yogurt" con self-service, en la ciudad de Esmeraldas; por la Pontificia Universidad Católica del Ecuador. El proyecto se distingue porque los 
clientes eligen la cantidad de producto y envase que ellos deseen, y así pagar según sus elecciones. El impacto general que se encontró en este plan de negocios fue positivo (Del Castillo Mera, 2016).

Además, se consideró el plan de negocios desarrollado en la Universidad Internacional del Ecuador, para elaboración de helados de quinua, en la ciudad de Quito, sector Villaflora. Los resultados proyectados son positivos desde el punto de vista económico financiero, con ingresos en el orden de $\$ 271.439,00$ a partir del quinto año. (Asanza Espinosa, 2017).

Otro proyecto considerado fue el plan de negocios para la elaboración y comercialización de helado congelado con nitrógeno líquido en la ciudad de Quito, en la Universidad de las Américas. Lo distintivo del proyecto es que el nitrógeno líquido permite que los helados se hagan en el instante, quedando más cremosos y suaves; no se requieren muchas neveras o congeladores. (Gaibor Espín, Estupiñan, \& Alexandra, 2016). Finalmente, resultó interesante revisar un plan de negocios que en la producción de los helados utilizan como base al maíz.
Fue desarrollado en la Universidad Politécnica Salesiana-Sede Quito. En sus conclusiones se encontró: que el Maíz- ice tendría un alto índice de aceptación tanto para su consumo como para su comercialización, lo que se reflejó mediante el análisis de estudio de mercado, pues del total de la muestra el 82,5\% estaría dispuesto a consumir este producto (Chasipanta, Nathaly, Gualoto, \& Nathaly, 2012).

El mercado de alimentos especiales es un nicho de mercado basado en la generación de productos alimenticios que sean beneficiosos para el bienestar y para sobrellevar alguna enfermedad 0 intolerancia. Este tipo de alimentos abarcan las siguientes características:

- Naturally Healthy (NH): Naturalmente contiene substancia que mejora la salud y el bienestar.

- Better For You (BFY): Reducido en azúcar, reducido en carbohidratos, grasa, sal.

- Food Intolerance: Sin lactosa, sin gluten, para diabéticos.

- Organic: Certificado orgánico por un organismo aprobado de certificación. 
- Fortified/Functional (FF): Ingredientes añadidos para mejorar funciones del cuerpo (calcio, vitaminas, omega-3).

El emprendimiento conjuga la experiencia de proyectos de producción de los helados artesanales con el mercado de alimentos especiales, lo cual se valora su sostenibilidad comercial, económica y financiera a través del Plan de Negocios

\section{Metodología (materiales y métodos)}

Se utilizó la metodología de sistematización de experiencias de emprendimiento y la utilización de los métodos de investigación de mercado, a través de la obtención de información primaria con la realización de encuestas y entrevistas a los actores claves relacionados con el objeto del proyecto.

Se utilizaron las técnicas de encuesta, las cuales se aplicaron a los potenciales consumidores del producto, para determinar el nivel de aceptación en el mercado, sus necesidades, gustos, etc. Se aplicó un cuestionario considerando la población de la ciudad de Portoviejo, para el cálculo de la muestra, se incluyó tanto la parte urbana como rural. De acuerdo con el Instituto Nacional de Estadística y Censos (2010) la población es de 280.029 habitantes. La muestra determinada fue de 384 personas, aplicando la siguiente fórmula:

$$
\begin{aligned}
& \mathrm{n}=((\llbracket Z) \rrbracket \wedge 2 \quad(p)(q) N) /(\llbracket(Z) \rrbracket \wedge 2 \\
& (p)(q)+N(\llbracket e) \rrbracket \wedge 2)
\end{aligned}
$$

En donde:

$Z=$ nivel de confianza $=1.96$

$\mathrm{p}=$ Probabilidad de ocurrencia .

$q=$ Probabilidad de no ocurrencia .

N= Población

$\mathrm{e}=$ Error de muestreo: $5 \%$

Según el Gobierno provincial de Manabí, Portoviejo posee 14 parroquias, de las cuales se seleccionaron 4 de estas parroquias para la aplicación de las encuestas. Las parroquias fueron: Parroquia Portoviejo, 12 de marzo, Andrés de Vera y Simón Bolívar. 


\section{Resultados (análisis e interpretación de los resultados)}

La misión de la empresa que se deriva del emprendimiento es ser una entidad dedicada a la elaboración y comercialización de helados sin ingredientes de origen animal o sus derivados, siempre apuntando a satisfacer las diversas necesidades en gustos de sabores, con un nivel satisfactorio de calidad.

Se aspira a ser una de las heladerías líderes en calidad y servicio en la ciudad de Portoviejo, satisfaciendo las necesidades de nuestros clientes $\mathrm{y}$ las necesidades internas de nuestra empresa, manteniendo a la vez una imagen fresca y dinámica a través de la innovación y diferenciación de nuestros productos acorde a las necesidades de los segmentos identificados.

El nombre de la empresa es "Chikeu" (nombre y apodo de la representante legal), y el slogan es "vegan ice cream", el cual fue seleccionado para darle al consumidor información a primera instancia de las características del producto.

\section{- Plan de mercadotecnia}

La puesta en escena es muy importante para el éxito de una heladería. Hay que escoger la decoración más adecuada para que el cliente se sienta a gusto y cuidar los aspectos de limpieza, colores, olores y visuales. Por ejemplo, resulta atractivo que el obrador esté a la vista y que la vitrina exterior presente los helados también a la altura de la mirada de los más pequeños - muchas veces, los mejores clientes -. Aun así, las mejores heladerías, ya consolidadas, pueden decidir sacrificar la visibilidad de los helados, guardándolos en los tubos de glicol (pozzetti), para una mayor conservación y calidad.

El boca a boca es la mejor arma para atraer a más clientes y es necesario trabajar la fidelización con la calidad y la simpatía. Pero también se aprecian mucho los detalles sorpresivos, que en algunos casos constituyen la seña distintiva del establecimiento. Algunos ejemplos: el cono relleno del chocolate de una fuente, la forma de flor que adquiere el helado al ser servido, un sándwich helado, un cucurucho con forma de 
pez, etc. También habrán de emplearse medios y canales de comunicación, tanto offline (el buzoneo o reparto de propaganda, por ejemplo), como online (página web y redes sociales, sobre todo Instagram y Facebook).

\section{Producto}

La tendencia del mercado es que el helado artesanal se percibe como saludable. Para estar al paso de las tendencias de consumo más actuales, muchas heladerías introducen variantes tales como la leche sin lactosa, la miel en vez del azúcar, los toppings de fruta fresca e incluso cuentan con una sección bio o vegana. Sin duda, en este negocio, es necesario adaptar elementos de la oferta para satisfacer a una clientela muy variada, compuesta por niños, personas mayores, turistas, locales, extranjeros, etc. $Y$ para deleitar a la gran mayoría y diferenciarse de los competidores, la calidad de los helados representa la mayor significación.

El segmento de helados en Ecuador es un mercado en crecimiento, más aún si se compara con el mercado latinoamericano; en otros países el consumo per cápita es mayor. En el caso de Chile, donde el consumo promedio de helado es 6 litros por persona al año, seguido de Brasil, cuyo indicador es 4 litros por persona; no así en Ecuador donde el promedio es alrededor de 1.9; nivel que demuestra que hay una gran oportunidad para hacer que se eleven los niveles de consumo. Para ello se requiere ofrecer al consumidor nuevas alternativas, no simplemente mostrar los formatos regulares, sino nuevas alternativas de consumo e innovación en los helados.

De acuerdo con los resultados obtenidos para el proyecto, a través de las encuestas que se le aplicó al público objetivo, se pudo encontrar información relevante la cual brinda aportes importantes para la determinación de las características del producto y las cuales van a ser incorporadas. También se pudo confirmar la aceptación del producto por parte del consumidor por los helados veganos de tipo artesanal, es decir están dispuestos a probar algo diferente, siempre y cuando sean de calidad. Los sabores identificados por la preferencia son: coco, mango, banana, piña y fresa. 
El helado es de tipo artesanal, elaborado a base de frutas con complementos como la mantequilla de maní, leche de almendra y de coco. Se comenzará con 9 sabores, a los cuales se les podrá poner toppings al gusto del cliente. Es importante que tanto las bases del helado, como sus complementos y toppings no llevaran ningún tipo de ingredientes de origen animal (vegano). Los helados van a ser comercializados a través de tres presentaciones, de cono, canasta de galleta y toppings, donde los sabores van de acuerdo a la elección de los clientes.

La primera presentación es en cono, donde el cliente podrá elegir 2 sabores. El segundo tipo de presentación es en canastitas de galleta, donde se colocará el producto ( 3 sabores) y después se podrán agregar 2 toppings. El tercer tipo de presentación (helado para comer en el camino) va a ser por medio de un envase biodegradable, - también por medio del propio envase que el cliente traiga además de su cuchara, lo que se busca con esto es educar al consumidor para reducir la utilización de plástico y cuidar el medio ambiente.
Las condiciones de abastecimiento de insumos garantizarán a través del establecimiento de alianzas y relaciones a mediano plazo con los productores agrícolas. De manera que se garantice el abastecimiento satisfactorio de la materia prima, considerando: precios, garantías, descuentos por cantidad, calidad, tiempo de entrega, así como las formas y medios de pago.

\section{Precio}

Para determinar el precio del producto se tiene en cuenta, por una parte, el análisis de los costos y gastos; y además se analiza el precio de referencia de la competencia. De manera que se pueda seguir una estrategia de introducción y posteriormente de mantenimiento y consolidación ante la competencia. En el análisis competitivo de referencia, se consideraron los precios de los precios de los principales competidores del mercado de helados, y también de acuerdo a las preferencias de heladerías expresadas por las personas encuestadas. En la siguiente tabla se relacionan las principales heladerías y el precio de algunas presentaciones de sus productos: 
Tabla 1. Análisis de precios de referencia del mercado.

\begin{tabular}{|l|c|c|c|c|c|}
\hline & \multicolumn{5}{|c|}{ HELADERÍAS } \\
\hline $\begin{array}{c}\text { PRESENTACIONES } \\
\text { DEL PRODUCTO }\end{array}$ & REDBERRY & GOYOGU & NICECREAM & $\begin{array}{c}\text { PEPE } \\
\text { TUCAN }\end{array}$ & GREENFROST \\
\hline Helado con Cono & $\$ 1.00$ & $\$ 0.90$ & $\$ 0.90-\$ 2.25$ & $\$ 1.00$ y $\$ 2.75$ & $\$ 1.00$ \\
\hline $\begin{array}{l}\text { Helado con } \\
\text { Toppings }\end{array}$ & $\$ 1.50-\$ 2.75$ & $\begin{array}{c}\$ 1.50- \\
\$ 3.00\end{array}$ & $\$ 1.25-\$ 2.90$ & $\$ 1.25-\$ 2.75$ & $\$ 1.50-\$ 3.00$ \\
\hline $\begin{array}{l}\text { Presentación en } \\
\text { canastilla de galleta }\end{array}$ & $*$ & $*$ & $\$ 1.70$ y $\$ 2.60$ & $*$ & $*$ \\
\hline
\end{tabular}

Fuente: Precio de las principales heladerías de la ciudad de Portoviejo (análisis competitivo).

Se observa que el precio promedio de los helados de cono sencillos es de $\$ 0.90-\$ 1.00$, y los más complejos es de más de $\$ 1.00$ hasta llegar a los $\$ 2.75$. El precio de los helados de toppings va desde $\$ 1.25$ a los $\$ 3.00$. $Y$ los conos de galleta donde solo los ofrece NICECREAM hay dos presentaciones de dos tamaños, donde la primera tiene un valor $\$ 1.70$ y la segunda un valor de $\$ 2.60$. Es importante mencionar que dentro del mercado de las heladerías la de mayor reputación y competitividad de precios es Greenfrost, además de que es la que posee más sucursales dentro de la ciudad.

Para la presentación en conos, el establecimiento de un precio de $\$ 1.00$, es coherente con el rango de precios con el cual se mueve la competencia, estableciéndose una mayor diferenciación al añadirle un mayor valor agregado al producto. A continuación, se presenta la tabla 2 , donde se detallaría como quedarían los precios a primera estancia según el análisis competitivo:

Tabla 2. Precio en primera estancia de presentaciones del producto

\begin{tabular}{|c|c|c|c|c|}
\hline \multicolumn{5}{|c|}{ PRECIO EN PRIMERA ESTANCIA DE PRESENTACIONES DEL PRODUCTO } \\
\hline DESCRIPCIÓN & \multicolumn{3}{|c|}{ PRECIO } & IMAGEN \\
\hline Presentación en cono. & \multicolumn{3}{|c|}{$\$ 1.00$} & \\
\hline \multirow{2}{*}{$\begin{array}{l}\text { Presentación helada con } \\
\text { toppings. }\end{array}$} & $\$ 1.50$ & $\$ 2.00$ & $\$ 2.50$ & \\
\hline & Pequeño & Mediano & Grande & \\
\hline $\begin{array}{c}\text { Presentación en canastilla } \\
\text { de galleta }\end{array}$ & \multicolumn{3}{|c|}{$\$ 2.50$} & \\
\hline
\end{tabular}




\section{Plaza}

La plaza o distribución es el instrumento del Marketing Mix que relaciona a los productores de un bien o servicio con los consumidores de este. Permite que los distintos productos estén al alcance de los consumidores en el momento, forma y lugares apropiados. La comercialización del producto se ubica en la parte céntrica de la ciudad de Portoviejo, en la calle Rocafuerte y 10 de agosto (esquina), por lo que el canal de distribución va a ser directamente con el consumidor final (B2C).

Los motivos principales por lo que se seleccionó la ubicación ya detallada con anterioridad son:
- La ubicación está en toda la parte céntrica de la ciudad, por lo que una gran cantidad de personas pasa por ahí. Esto ayudará a la captación de clientes.

- El terreno donde se plantea poner la edificación es heredado a la representante legal del negocio.

\section{$>$ Promoción}

El lanzamiento del emprendimiento se realizará a través del marketing online y marketing offline; en la tabla 3 se resume las acciones respectivamente. Se consideran además las alianzas con centros diabetológicos y cardiológicos.

Tabla 3. Marketing de lanzamiento

\begin{tabular}{|c|c|c|}
\hline \multicolumn{3}{|c|}{ MARKETING DE LANZAMIENTO } \\
\hline TIPO DE MARKETING & DETALLE & costo \\
\hline Marketing online & $\begin{array}{l}\text { - Creación y publicidad a través de una } \\
\text { página de Facebook e Instagram. }\end{array}$ & $\$ 30$ \\
\hline Marketing offline & $\begin{array}{l}\text { - Se invitará al público a conocer la } \\
\text { heladería a través de la entrega de } \\
\text { afiches informativos. Los responsables } \\
\text { serán dos promotores, cuyos puntos } \\
\text { estratégicos de entrega serán los } \\
\text { centros educativos de la ciudad de } \\
\text { Portoviejo y las calles de la parte } \\
\text { céntrica de la ciudad. Los dos centros } \\
\text { educativos son: la Universidad Técnica } \\
\text { de Manabí y el Colegio Portoviejo. } \\
\text { Realizar visitas a los principales } \\
\text { centros diabetológicos y cardiológicos } \\
\text { de la ciudad de Portoviejo para } \\
\text { establecer un diálogo directo para } \\
\text { promocionar el producto en estos } \\
\text { centros y obtener posibles convenios. }\end{array}$ & $\$ 100$ \\
\hline
\end{tabular}




\begin{tabular}{|l|l|}
\hline Se llevará muestras de diferentes \\
sabores del producto con su respectiva \\
valoración nutricional adecuada para \\
los distintos tipos de pacientes. Se \\
dejará en estos centros tarjetas de \\
presentación para el posterior contacto.
\end{tabular}

Fuente: María Eulalia Moreira Mera (2021).

Previamente se desarrollará una campaña educativa y cultural a través de las redes sociales orientada al consumo de helados veganos. Se debe mostrar al público los beneficios del producto, su valor nutricional y principalmente sus ventajas para las personas con intolerancia a la lactosa, diabetes y problemas cardiovasculares.

\section{Descripción del proceso de producción}

El proceso de producción consta de las siguientes actividades:

- Recepción de la materia prima: Se recibe la materia prima (frutas) por parte de los agricultores.

- Verificación del peso: Después se procede en pesarla en la balanza digital para comprobar que la cantidad entregada sea la acordada. Si el peso entregado de la materia prima no es el acordado, se hace el reclamo y se espera nuevamente a la recepción de la materia prima faltante.

- Verificación de la calidad: Este paso consiste en verificar que todas las frutas no tengan algún desperfecto que afecte su calidad. Si la calidad es inadecuada, se hace un reclama al proveedor, se devuelve la materia prima recibida, y se regresa a la recepción de una nueva materia prima.

- Lavado: Se procede a lavar muy bien la materia prima para evitar cualquier contaminación.

- Pelado: Una vez limpia la materia prima, se pela la fruta y se cortan las hojas.

- Cortado: Una vez la fruta este en sin cascara o hojas, se la corta en porciones.

- Empaquetado: Se la coloca cada tipo de fruta en recipientes diferentes. 
- Almacenamiento

congelación:

La

empaquetada se la coloca en el congelador para que entre en estado congelado.

- Procesamiento: Según el pedido del cliente (sabor del helado), se sacan las frutas para procesarlas en la máquina; además que al mismo tiempo se la va colocando en el cono o envase.

- Adherir Toppings: Se colocan los toppings según la preferencia del cliente.
- Entrega del producto: Se entrega el producto ya listo para degustar.

\section{Plan económico financiero}

El monto total de inversión es de $\$ 5297$, de ello el $17 \%$ corresponde a equipos, $62 \%$ a muebles y equipo de oficina $y$ el $21 \%$ al Capital de Trabajo; este último se determinó a partir de garantizar la cobertura financiera para tres meses de gastos operativos del primer año.

Tabla 4. Inversión.

\begin{tabular}{|l|r|r|r|}
\hline Inversión & \multicolumn{2}{|c|}{ Total } \\
\hline Maquinaria y equipo & Cantidad & Precio Unitario & 340 \\
\hline Máquina de helados (FruitiFree-z) & 4 & 85 & 102 \\
\hline Licuadora industrial. & 2 & 51 & 401 \\
\hline Congelador. & 1 & 401 & 45 \\
\hline Balanza electrónica. & 1 & 45 & $\mathbf{8 8 8}$ \\
\hline Total Equipos & & & $\mathbf{3 , 2 8 9}$ \\
\hline Muebles y equipo de Oficina & & & $\mathbf{1 , 1 2 0}$ \\
\hline Capital de trabajo & & $\mathbf{5 , 2 9 7}$ \\
\hline Monto de Inversiòn & & & \\
\hline
\end{tabular}

Fuente: Autora (2021).

\section{Proyección de ventas}

Las previsiones estimadas de ventas, a partir del análisis del mercado y el segmento que se va a atender, parte de la estructura del producto en: conos, canasta y helado de envase; así como la cantidad estimada mensual y anual. Se toman los precios determinados con anterioridad, con referencia a la competencia actual. La estimación de ventas para los cinco años en que se evalúa el proyecto es la siguiente: 
Tabla 5. Proyección de ventas.

\begin{tabular}{|l|r|r|r|r|r|}
\hline \multicolumn{1}{|c|}{ Conceptos UM: } & \multicolumn{1}{c|}{ año 1 } & \multicolumn{1}{c|}{ año 2 } & \multicolumn{1}{c|}{ año 3 } & \multicolumn{1}{c|}{ año 4 } & \multicolumn{1}{c|}{ año 5 } \\
\hline Ventas Totales & $\mathbf{4 2 , 5 0 4}$ & $\mathbf{4 3 , 3 5 4}$ & $\mathbf{4 4 , 2 2 1}$ & $\mathbf{4 5 , 1 0 6}$ & $\mathbf{4 6 , 0 0 8}$ \\
\hline Cono & 10,080 & 10,282 & 10,487 & 10,697 & 10,911 \\
\hline Canasta de galleta & 12,600 & 12,852 & 13,109 & 13,371 & 13,639 \\
\hline Helado envase & $\mathbf{1 9 , 8 2 4}$ & $\mathbf{2 0 , 2 2 0}$ & 20,625 & 21,037 & 21,458 \\
\hline Grande & 10,080 & 10,282 & 10,487 & 10,697 & 10,911 \\
\hline Mediano & 4,704 & 4,798 & 4,894 & 4,992 & 5,092 \\
\hline Pequeño & 5,040 & 5,141 & 5,244 & 5,348 & 5,455 \\
\hline
\end{tabular}

Fuente: Autora (2021).

Para la proyección hasta el año 5 se estimó un incremento anual del $2 \%$, con un criterio conservador, que se sitúa por debajo del crecimiento del mercado.

\section{Costos y Gastos}

Se determinaron los costos directos de materias primas y materiales de acuerdo con la norma de consumo y el precio unitario, según negociación con los proveedores previstos. Las materias primas con mayor valor dentro de los costos son: la leche de almendra y de coco, la mantequilla de cacahuete, el oreo $y$ as almendras.
La mano de obra representa el $53 \%$ de los costos y gastos, lo que es coherente con un negocio artesanal. Administrativos. Al nivel administrativo, está el gerenteadministrador de la empresa, responsable de la empresa legalmente y de tomar las decisiones en el negocio, capacitación y manejo del personal.

El nivel auxiliar de apoyo se vincula con los servicios contable financieros a contratar. A nivel operativo, se cuenta con 2 personas, relacionadas con las ventas y la producción respectivamente.

Tabla 6. Costos y gastos.

\begin{tabular}{|l|r|r|r|}
\hline Costos y Gastos & Mensual & Anual & Estructura \\
\hline Directos & 280 & 3,360 & $16 \%$ \\
\hline Mano obra & 917 & 11,004 & $53 \%$ \\
\hline Servicios y Mtto & 100 & 1,200 & $6 \%$ \\
\hline Depreciaciòn & 39 & 462 & $2 \%$ \\
\hline Gastos Direcciòn & 404 & 4,848 & $23 \%$ \\
\hline Total de costos & $\mathbf{1 7 4 0}$ & $\mathbf{2 0 , 8 7 4}$ & $\mathbf{1 0 0 \%}$ \\
\hline
\end{tabular}

Fuente: Autora (2021). 


\section{Evaluación económica financiera}

La evaluación del proyecto considero en primer lugar el proyecto puro sin financiamiento, y posteriormente se le incorporo los flujos del financiamiento actualizado con el costo medio ponderado de capital.
Sobre la base de los supuestos tomados para la proyección y considerando un nivel de costos que permiten márgenes de ganancia de explotación como promedio de más del $50 \%$ por peso de ventas, permite generar un Valor Actual Neto positivo y una tasa interna de retorno que soporta niveles mayores de costo de capital.

Tabla 7. Flujo de Caja del proyecto.

\begin{tabular}{|c|c|c|c|c|c|c|}
\hline \multicolumn{7}{|c|}{ Flujo de Caja sin financiamiento } \\
\hline Conceptos & 0 & 1 & 2 & 3 & 4 & 5 \\
\hline Inversión & $-5,297$ & & & & & \\
\hline Ventas & & 42,504 & 43,354 & 44,221 & 45,106 & 46,008 \\
\hline Costos material directo & & 3,360 & 3,626 & 3,912 & 4,221 & 4,555 \\
\hline Costo mano de obra & & 11,004 & 11,004 & 11,004 & 11,004 & 11,004 \\
\hline Costos indirectos & & 1,200 & 1,234 & 1,269 & 1,305 & 1,341 \\
\hline Depreciación & & 462 & 462 & 462 & 462 & 462 \\
\hline Gasto administración & & 4,848 & 4,848 & 4,848 & 4,848 & 4,848 \\
\hline BAT & & 21,630 & 22,180 & 22,726 & 23,265 & 23,797 \\
\hline Impuesto & & 7,570 & 7,763 & 7,954 & 8,143 & 8,329 \\
\hline $\mathrm{BN}$ & & 14,059 & 14,417 & 14,772 & 15,123 & 15,468 \\
\hline Flujo de caja & $-5,297$ & 14,522 & 14,879 & 15,234 & 15,585 & 15,930 \\
\hline VAN & 45,429 & & & & & \\
\hline TIR & $276.2 \%$ & & & & & \\
\hline PR actualizado & 0.50 & & & & & \\
\hline meses & 6 & & & & & \\
\hline
\end{tabular}

Fuente: Autora (2021).

\section{Flujo de Caja con financiamiento}

Se va a financiar el $60 \%$ de la inversión con crédito bancario, a través del BAN Ecuador, cuya tasa de interés es de $11.86 \%$ para financiamientos destinados al emprendimiento. El plazo de vencimiento es de dos años, con vencimiento trimestral a través de cuotas fijas. Las cuotas fijas de pago trimestral son de $\$ 439$, que se incorporan al flujo de caja, con salidas anuales por $\$ 1756$. En la siguiente tabla se determina por períodos el pago de intereses y principal. 
Tabla 7. Amortización del crédito.

\begin{tabular}{|c|r|r|r|r|}
\hline Períodos & \multicolumn{1}{|l|}{ Monto } & \multicolumn{1}{l|}{ Interes } & \multicolumn{1}{l|}{ Pago } & \multicolumn{1}{l|}{ principal } \\
\hline 1 & 3178 & 94.23 & $-\$ 452$ & $-\$ 357.85$ \\
\hline 2 & $\$ 2,820$ & 83.62 & $-\$ 452$ & $-\$ 368.46$ \\
\hline 3 & $\$ 2,452$ & 72.70 & $-\$ 452$ & $-\$ 379.39$ \\
\hline 4 & $\$ 2,072$ & 61.45 & $-\$ 452$ & $-\$ 390.64$ \\
\hline 5 & $\$ 1,682$ & 49.87 & $-\$ 452$ & $-\$ 402.22$ \\
\hline 6 & $\$ 1,280$ & 37.94 & $-\$ 452$ & $-\$ 414.15$ \\
\hline 7 & $\$ 865$ & 25.66 & $-\$ 452$ & $-\$ 426.42$ \\
\hline 8 & $\$ 439$ & 13.02 & $-\$ 452$ & $-\$ 439.07$ \\
\hline
\end{tabular}

Fuente: Autora (2021).

El flujo de caja con financiamiento expresa el efecto del apalancamiento financiero al considerarse la actualización con un costo de capital medio ponderado del $10.63 \%$, lo que conduce a un incremento del VAN por $\$ 6284$, como se observa en la siguiente tabla

Tabla 8. Flujo de caja con financiamiento.

\begin{tabular}{|c|c|c|c|c|c|c|}
\hline Conceptos & 0 & 1 & 2 & 3 & 4 & 5 \\
\hline Inversión & $-2,119$ & & & & & \\
\hline Ventas & & 42,504 & 43,354 & 44,221 & 45,106 & 46,008 \\
\hline Costos material directo & & 3,360 & 3,626 & 3,912 & 4,221 & 4,555 \\
\hline Costo mano de obra & & 11,004 & 11,004 & 11,004 & 11,004 & 11,004 \\
\hline Costos indirectos & & 1,200 & 1,234 & 1,269 & 1,305 & 1,341 \\
\hline Depreciación & & 462 & 462 & 462 & 462 & 462 \\
\hline Gasto administración & & 4,848 & 4,848 & 4,848 & 4,848 & 4,848 \\
\hline BAIT & & 21,630 & 22,180 & 22,726 & 23,265 & 23,797 \\
\hline Intereses & & 312 & 126 & & & \\
\hline BAT & & 21,318 & 22,054 & 22,726 & 23,265 & 23,797 \\
\hline Impuesto & & 7,570 & 7,763 & 7,954 & 8,143 & 8,329 \\
\hline $\mathrm{BN}$ & & 14,059 & 14,417 & 14,772 & 15,123 & 15,468 \\
\hline Pago de principal & & $-1,496$ & $-1,682$ & & & \\
\hline Flujo de caja & $-2,119$ & 13,025 & 13,198 & 15,234 & 15,585 & 15,930 \\
\hline VAN & 51,713 & & & & & \\
\hline TIR & $617.8 \%$ & & & & & \\
\hline
\end{tabular}

Fuente: Autora (2021).

\section{Conclusiones}

Se deriva del análisis realizado que para pequeños proyectos resulta válido elaborar planes de negocios adaptados a sus particularidades, que permita superar las barreras de la falta de preparación, la improvisación al decidir implementar una idea de negocio; así como la limitación de recursos materiales y financieros. 
Se concluye que el proyecto es factible desde el punto de vista comercial. al determinarse que existe un significativo grado de aceptación por parte del segmento de mercado al que va dirigido el producto. Los resultados de los indicadores para evaluar la factibilidad del proyecto son satisfactorios, considerando una tasa interna de retorno muy superior al costo de oportunidad para un proyecto similar y del costo financiero del sistema bancario

Los riesgos del proyecto están vinculado a las particularidades naturales de la agricultura, lo cual se integran a los riesgos propios de la comercialización en el mercado. De esta manera hay que lograr procesos de diversificación que eviten la concentración del negocio en pocos productos agrícolas y la vigilancia de la competencia, así como del comportamiento del consumidor

\section{Bibliografía}

Asanza Espinosa, V. D. (2017). Plan de negocios para la elaboración de helados de quinua, en la ciudad de Quito, sector Villaflora, período: 2016 (Bachelor's thesis, Quito/UIDE/2017).
Chasipanta, E., Nathaly, T., Gualoto, Q., \& Nathaly, D. (2012). Proyecto de factibilidad para la creación de una microempresa productora $y$ comercializadora de helado de máiz, ubicada en la ciudad de Quito.

Codex Alimentarius. (2014). www.codexalimentarius.org. Obtenido:

http://www.saborysalud.com/ content/articles/866/1/Conozc a-los-diferentes-tiposdehelados/Page1.html

Del Castillo Mera, M. M. (2016). Estudio de factibilidad para la puesta en marcha de una heladería dedicada a la producción y comercialización de helados de yogurt con frutas "ICE FRUIT YOGURT" con self-service en la ciudad de Esmeraldas

Gaibor Espín, V. A., Estupiñan, F., \& Alexandra, P. (2016). Plan de negocios para la elaboración y comercialización de helado congelado con nitrógeno líquido en la ciudad de Quito (Bachelor's thesis, Quito: Universidad de las Américas, 2016.).

Instituto Nacional de Estadística y Censos. (2018). Encuesta Nacional De Empleo, Desempleo y Subempleo (ENEMDU). Obtenido de Instituto Nacional de 
Estadística y Censos:

http://www.ecuadorencifras.g ob.ec/documentos/nec/EMPL EO/2018/Septiembre-

2018/092018_Mercado\%20L aboral.pdf

Moreira Mera, M.E. (2019). Tesis pregrado plan de negocios para la creación de una heladería vegana en el cantón Portoviejo.

Pérez, J., \& Merino, M. (2010). Definición de Vegano. Obtenido de Definición: https://definicion.de/vegano/ 